\title{
HOMOGENEOUS COUNTABLE CONNECTED HAUSDORFF SPACES
}

\author{
JOSEPH M. MARTIN
}

In 1925, P. Urysohn gave an example of a countable connected Hausdorff space [4]. Other examples have been contributed by $R$. Bing [1], M. Brown [2], and E. Hewitt [3]. Relatively few of the properties of such spaces have been examined. In this paper the question of homogeneity is studied. Theorem I shows that there exists a bihomogeneous countable connected Hausdorff space. Theorems II and III deal with other questions related to homogeneity.

A space $Z$ is homogeneous if and only if for every pair of elements $x$ and $y$ of $Z$ there exists a homeomorphism $f$ of $Z$ onto itself such that $f(x)=y$. A space $Z$ is bihomogeneous if and only if for every pair of elements $x$ and $y$ of $Z$ there exists a homeomorphism $f$ of $Z$ onto itself such that $f(x)=y$ and $f(y)=x$.

THEOREM I. There exists a bihomogeneous countable connected Hausdorff space.

Proof. Let $X_{0}$ be a countable connected Hausdorff space $\left\{x_{1}^{0}, x_{2}^{0}, \cdots\right\}$ and let $\left\{X_{1}, X_{2}, \cdots\right\}$ be a countably infinite family of mutually disjoint homeomorphic images of $X_{0}$. For each $i$, let $h_{i}$ be a homeomorphism of $X_{0}$ onto $X_{i}$, and for each $i$ and $j$, let $x_{j}^{i}$ be $h_{i}\left(x_{j}^{0}\right)$.

Let $p_{1}^{1}$ be $\left(x_{1}^{2}, x_{2}^{1}\right)$, and $p_{2}^{1}$ be $\left(x_{1}^{1}, x_{2}^{2}\right)$. Let $p_{1}^{2}$ be $\left(x_{1}^{2}, x_{2}^{1}, x_{3}^{3}\right), p_{2}^{2}$ be $\left(x_{1}^{1}, x_{2}^{2}, x_{3}^{4}\right), p_{3}^{2}$ be $\left(x_{1}^{4}, x_{2}^{3}, x_{3}^{1}\right)$, and $p_{4}^{2}$ be $\left(x_{1}^{3}, x_{2}^{4}, x_{3}^{2}\right)$. Suppose that $n$ is a positive integer such that if $i \leqq 2^{n}$, (1) $p_{i}^{n}$ exists and (2) for some positive integers $k_{1}, k_{2}, \cdots$, and $k_{n+1}, p_{i}^{n}=\left(x_{1}^{k_{1}}, x_{2}^{k_{2}}, \cdots, x_{n+1}^{k_{n+1}}\right)$. Then let $p_{i}^{n+1}$ be $\left(x_{1}^{k_{1}}, x_{2}^{k_{2}}, \cdots, x_{n+1}^{k_{n+1}}, x_{n+2}^{i+2^{n}}\right)$ and $p_{i+2^{n}}^{n+1}$ be

$$
\left(x_{1}^{k_{1}+2^{n}}, x_{2}^{k_{2}+2^{n}}, \cdots, x_{n+1}^{2^{n}+k_{n+1}}, x_{n+2}^{i}\right) \text {. }
$$

For each $n$ let $Z_{n}$ be $\bigcup_{i=1}^{2^{n}}\left(\left[X_{i}-\bigcup_{j=1}^{n+1}\left\{x_{j}^{i}\right\}\right] \cup\left\{p_{i}^{n}\right\}\right)$. A neighborhood system for $Z_{n}$ is defined as follows: If $z$ is a point of some $X_{i}$, then $\Re(z)$ denotes the neighborhood system of $z$ in $X_{i}$. If $z \in \cup_{i=1}^{2^{n}}\left[X_{i}-\bigcup_{j=1}^{n+1}\left\{x_{j}^{i}\right\}\right]$, then for some $i$ and $j, i \leqq 2^{n}$ and $j>n+1, z$ $=x_{j}^{i}$, and in this case the neighborhood system $N^{n}(z)$ of $z$ in $Z_{n}$ is $\left\{U: U \in \Re(z)\right.$ and $\left.\left[U_{j=1}^{n+1}\left\{x_{j}^{i}\right\}\right] \cap U=\varnothing\right\}$. Suppose that $k \leqq 2^{n}$ and $k_{1}, k_{2}, \cdots$, and $k_{n+1}$ are positive integers such that

Presented to the Society, January 29, 1960; received by the editors April 26, 1960. 


$$
p_{k}^{n}=\left(x_{1}^{k_{1}}, x_{2}^{k_{2}}, \cdots, x_{n+1}^{k_{n+1}}\right) \text {. }
$$

If $i \leqq n+1$, let $G_{k_{i}}$ be $\left\{U: U \in \Re\left(x_{i}^{k_{i}}\right)\right.$ and if $r \neq i$ and $r \leqq n+1$, then $\left.x_{r}^{k_{i}} \notin U\right\}$. Then the neighborhood system $N^{n}\left(p_{k}^{n}\right)$ of $p_{k}^{n}$ in $Z_{n}$ is $\left\{\left[U_{i=1}^{n+1}\left(U_{i}-\left\{x_{i}^{k_{i}}\right\}\right)\right] \cup\left\{p_{k}^{n}\right\}: U_{i} \in G_{k_{i}}\right\}$.

If $n$ is a positive integer, $k \leqq 2^{n}$ and $p_{k}^{n}=\left(x_{1}^{k_{1}}, x_{2}^{k_{2}}, \cdots, x_{n+1}^{k_{n+1}}\right)$, then the points $x_{1}^{k_{1}}, x_{2}^{k_{2}}, \cdots$, and $x_{n+1}^{k_{n+1}}$ are the coordinates of $p_{k}^{n}$.

Lemma 1. For each $n, Z_{n}$ is a countable connected Hausdorff space.

Proof. Let $n$ be a positive integer. It is clear that $Z_{n}$ is a countable Hausdorff space. Suppose that $Z_{n}$ is not connected. Then there exist disjoint nonempty open sets $A$ and $B$ such that $A \cup B=Z_{n}$. For each $i, i \leqq 2^{n}$, let $K_{i}$ be $\left\{x_{j}^{i}: x_{j}^{i} \in Z_{n}\right\} \cup\left\{p_{k}^{n}: p_{k}^{n}\right.$ has a coordinate which belongs to $\left.X_{i}\right\}$. For each $i, K_{i}$ is connected since it is homeomorphic with $X_{i}$. Assuming that the notation is chosen so that $K_{1} \subset A$, let $j$ be $\min \left\{i: K_{i} \subset B\right\}$. Now there exist integers $k$ and $r, r<j$, such that $p_{k}^{n}$ has a coordinate belonging to $X_{r}$ and a coordinate belonging to $X_{j}$. Then $p_{k}^{n} \in K_{r} \cap K_{j}$ and hence $p_{k}^{n} \in A \cap B$. This contradicts the fact that $A \cap B=\varnothing$. This establishes the lemma.

Suppose that $n \leqq r, U_{n}$ is an open set in $Z_{n}$, and $U_{r}$ is an open set in $Z_{r}$. Then the notation

$$
U_{n} \widetilde{\subset} U_{r}
$$

means that (1) if $p_{j}^{n} \in U_{n}$, then $p_{j}^{r} \in U_{r}$ and (2) if $x_{s}^{t} \in U_{n}$ then either (i) $x_{z}^{z} \in U_{r}$ or (ii) for some $k, p_{k}^{r} \in U_{r}$ and $x_{z}^{z}$ is a coordinate of $p_{k}^{r}$.

Certain permutations are to be defined now. Let $q_{1}^{1}$ be the identity, and $q_{2}^{1}$ be $(1,2)$ each on $\{1,2\}$. Let $q_{1}^{2}$ be the identity, $q_{2}^{2}$ be $(1,2)(3,4)$, $q_{3}^{2}$ be $(1,3)(2,4)$, and $q_{4}^{2}$ be $(1,4)(2,3)$, each on $\{1,2,3,4\}$. Suppose that $n$ is a positive integer and if $i \leqq 2^{n}$, there is a permutation $q_{i}^{n}$ on $\left\{1,2, \cdots, 2^{n}\right\}$ such that for distinct positive integers $k_{1}, k_{2}, \cdots$, and $k_{2^{n}}, q_{i}^{n}=\left(k_{1}, k_{2}\right)\left(k_{3}, k_{4}\right) \cdots\left(k_{2^{n}-1}, k_{2^{n}}\right)$. Then if $i \leqq 2^{n}, q_{i}^{n+1}$ $=\left(k_{1}, k_{2}\right)\left(k_{3}, k_{4}\right) \cdots\left(k_{2^{n}-1}, k_{2^{n}}\right)\left(k_{1}+2^{n}, k_{2}+2^{n}\right)\left(k_{3}+2^{n}, k_{4}+2^{n}\right) \cdots$ $\left(k_{2^{n}-1}+2^{n}, k_{2^{n}}+2^{n}\right)$ and $q_{i+2^{n}}^{n+1}=\left(k_{1}, k_{2}+2^{n}\right)\left(k_{3}, k_{4}+2^{n}\right) \cdots$ $\left(k_{2^{n}-1}, k_{2^{n}}+2^{n}\right)\left(k_{1}+2^{n}, k_{2}\right)\left(k_{3}+2^{n}, k_{4}\right) \cdots\left(k_{2^{n}-1}+2^{n}, k_{2^{n}}\right)$. Notice that each permutation is the product of disjoint 2-cycles and if $r \leqq 2^{n}$ and $s \leqq 2^{n}$ then there is one and only one positive integer $i$ such that $q_{i}^{n}(r)=s$ and $q_{i}^{n}(s)=r$.

LEMMA 2. If $i \leqq 2^{n}, k \leqq 2^{n}$, and $p_{k}^{n}=\left(x_{1}^{k_{1}}, x_{2}^{k_{2}}, \cdots, x_{n+1}^{k_{n+1}}\right)$, then $p_{Q_{i}(k)}^{n}=\left(x_{1}^{q_{i}^{n}\left(k_{1}\right)}, x_{2}^{q_{i}^{n}\left(k_{2}\right)}, \cdots, x_{n+1}^{q_{n}^{n}\left(_{n+1}\right)}\right)$.

Proof. The result is clear if $n=1$. Assume the result holds for $n$ and suppose that $i \leqq 2^{n}, k \leqq 2^{n}$, and $p_{k}^{n}=\left(x_{1}^{k_{1}}, x_{2}^{k_{2}}, \cdots, x_{n+1}^{k_{n+1}}\right)$. If 
$j \leqq n+1$, then $q_{i}^{n+1}\left(k_{j}\right)=q_{i}^{n}\left(k_{j}\right), q_{i}^{n+1}\left(k_{j}+2^{n}\right)=q_{i}^{n+1}\left(k_{j}\right)+2^{n}, q_{i+2^{n}}^{n+1}\left(k_{j}\right)$ $=q_{i}^{n+1}\left(k_{j}\right)+2^{n}$, and $q_{i+2^{n}}^{n+1}\left(k_{j}+2^{n}\right)=q_{i}^{n+1}\left(k_{j}\right)$. Therefore, if $m=i+2^{n}$, then

$$
\begin{aligned}
& p_{q_{i}^{n+1}(k)}^{n+1}=\left(x_{i}^{q_{i}^{n+1}\left(k_{1}\right)}, \cdots, x_{n+1}^{q_{i}^{n+1}\left(k_{n+1}\right)}, x_{n+2}^{q_{i}^{n+1}\left(k+2^{n}\right)}\right), \\
& p_{q_{i}^{n+1}\left(k+2^{n}\right)}^{n+1}=\left(x_{i}^{q_{i}^{n+1}\left(k_{1}+2^{n}\right)}, \cdots, x_{n+1}^{q_{i}^{n+1}\left(2^{n}+k_{n+1}\right)}, x_{n+2}^{q_{i}^{n+1}(k)}\right),
\end{aligned}
$$

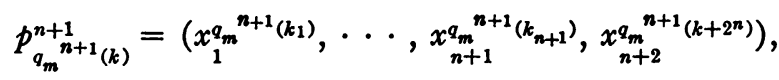

$$
\begin{aligned}
& p_{q_{m}{ }^{n+1}\left(k+2^{n}\right)}^{n+1}=\left(x_{1}^{q_{m}^{n+1}\left(k_{1}+2^{n}\right)}, \cdots, x_{n+1}^{q_{m}^{n+1}\left(2^{n}+k_{n+1}\right)}, x_{n+2}^{q_{m}^{n+1}(k)}\right) \text {. }
\end{aligned}
$$

This establishes Lemma 2.

For each $n$ and each $i, i \leqq 2^{n}$, let $g_{i}^{n}$ be the function from $Z_{n}$ onto $Z_{n}$ defined as follows: If $k \leqq 2^{n}$ and $j>n+1$, then $g_{i}^{n}\left(x_{j}^{k}\right)=x_{j}^{q_{i}^{n}}(k)$ and $g_{i}^{n}\left(p_{k}^{n}\right)=p_{l_{i}^{n}(\mathfrak{k})}^{n}$.

Lemma 3. For each $n$ and each $i, i \leqq 2^{n}, g_{i}^{n}$ is a homeomorphism of $Z_{n}$ onto $Z_{n}$.

Proof. Suppose that $i \leqq 2^{n}$. It is clear that $g_{i}^{n}$ is one-to-one and onto. Suppose that $k \leqq 2^{n}, j>n+1$ and $U \in N^{n}\left(x_{j}^{k}\right)$. It is clear that $g_{i}^{n}(U) \in N^{n}\left(g_{i}^{n}\left(x_{j}^{k}\right)\right)$. Suppose that $k_{1}, k_{2}, \cdots$, and $k_{n+1}$ are positive integers such that $p_{k}^{n}=\left(x_{1}^{k_{1}}, x_{2}^{k_{2}}, \cdots, x_{n+1}^{k_{n+1}}\right)$. Let $V$ be an element of $N^{n}\left(p_{k}^{n}\right)$. Then for each $r, r \leqq n+1$, there exists an element $V_{r}$ of $G_{k_{r}}$ such that $V=U_{r=1}^{n+1}\left(V_{r}-\left\{x_{r}^{k_{r}}\right\}\right) \cup\left\{p_{k}^{n}\right\}$. Now for each $r, r \leqq n+1$, $g_{i}^{n}\left(V_{r}\right) \in G_{q i}{ }^{n}\left(k_{r}\right)$. By Lemma 2,

$$
g_{i}^{n}\left(p_{k}^{n}\right)=\left(x_{1}^{q_{i}^{n}\left(k_{1}\right)}, \cdots, x_{n+1}^{q_{i}^{n}\left(k_{n+1}\right)}\right),
$$

and hence

$$
g_{i}^{n}(V)=\bigcup_{r=1}^{n+1}\left(g_{i}^{n}\left(V_{r}\right)-\left\{x_{r}^{q_{i}^{n}\left(k_{r}\right)}\right\}\right) \cup\left\{p_{q_{i}^{n}(k)}^{n}\right\}
$$

which is an element of $N^{n}\left(g_{i}^{n}\left(p_{k}^{n}\right)\right)$. This shows that $\left(g_{i}^{n}\right)^{-1}$ is continuous. Since $g_{i}^{n}=\left(g_{i}^{n}\right)^{-1}$ it follows that $g_{i}^{n}$ is a homeomorphism.

Lemma 4. For each $n, r$, and $s$ such that $r \leqq 2^{n}$ and $s \leqq 2^{n}$, there exists one and only one positive integer $i, i \leqq 2^{n}$, such that $g_{i}^{n}\left(p_{s}^{n}\right)=p_{r}^{n}$ and $g_{i}^{n}\left(p_{r}^{n}\right)=p_{s}^{n}$.

Proof. Suppose that $r \leqq 2^{n}$ and $s \leqq 2^{n}$. Now there exists one and only one positive integer $i, i \leqq 2^{n}$, such that $q_{i}^{n}(r)=s$ and $q_{i}^{n}(s)=r$. Then $g_{i}^{n}\left(p_{r}^{n}\right)=p_{q_{i}^{n}(r)}^{n}=p_{s}^{n}$ and $g_{i}^{n}\left(p_{s}^{n}\right)=p_{q_{i}^{n}(s)}^{n}=p_{r}^{n}$. 
For each $k$, let $p_{k}$ be the sequence whose $i$ th term is, for sufficiently large $n$, the $i$ th coordinate of $p_{k}^{n}$. Let $Z$ be $\left\{p_{k}: k\right.$ is a positive integer $\}$. A neighborhood system for $Z$ is defined as follows: If for some $i, U_{i}$ is an open set in $Z_{i}$ let $U_{i}^{*}$ denote the set of all points $p_{j}$ of $Z$ such that either (1) $p_{j}^{i} \in U_{i}$ or (2) $p_{j}$ has a coordinate in $U_{i}$. Now for each $k, N\left(p_{k}\right)$, the neighborhood system of $p_{k}$ in $Z$ is $\left\{\left(\cup_{i=n}^{\infty} U_{i}^{*}\right): k \leqq 2^{n}\right.$, $U_{i}$ is an open set in $Z_{i}, p_{k}^{i} \in U_{i}$, and $\left.U_{i} \widetilde{\subset} U_{i+1}\right\}$.

For each $r$, let $g_{r}$ be the function from $Z$ onto $Z$ defined as follows: Suppose that $k \leqq 2^{n}$. For some $s, s \leqq 2^{n}, g_{r}^{n}\left(p_{k}^{n}\right)=p_{s}^{n}$. Set $g_{r}\left(p_{k}\right)=p_{s}$. Then $g_{r}$ is well defined since if $t \geqq n, g_{r}^{t}\left(p_{k}^{t}\right)=p_{s}^{t}$.

It follows from Lemma 4 and the definition of $g_{i}$ that for each $r$ and each $s$ there exists one and only one positive integer $i$ such that $g_{i}\left(p_{r}\right)=p_{s}$ and $g_{i}\left(p_{s}\right)=p_{r}$.

Lemma 5. $Z$ is a countable connected Hausdorff space.

Proof. $Z$ is clearly a countable Hausdorff space. Suppose that $Z$ is not connected. Then there exist disjoint nonempty open sets $A$ and $B$ such that $A \cup B=Z$. There exist positive integers $k$ and $j$ such that $p_{k} \in A$ and $p_{j} \in B$. Let $n$ be a positive integer such that $k \leqq 2^{n}$ and $j \leqq 2^{n}$. Let $A_{n}$ be $\left\{p_{i}^{n}: p_{i} \in A\right\} \cup\left\{x_{i}^{s}: s \leqq 2^{n}\right.$ and there exists an element $p_{i}$ of $A$ such that $x_{i}^{s}$ is a coordinate of $\left.p_{i}\right\}$. Let $B_{n}$ be $\left\{p_{i}^{n}: p_{i} \in B\right\}$ $\cup\left\{x_{i}^{s}: s \leqq 2^{n}\right.$ and there exists an element $p_{i}$ of $B$ such that $x_{i}^{s}$ is a coordinate of $\left.p_{i}\right\}$. Now $A_{n}$ and $B_{n}$ are nonempty disjoint open sets in $Z_{n}$, and $A_{n} \cup B_{n}=Z_{n}$. This contradicts the fact that $Z_{n}$ is connected, and hence $Z$ is connected.

\section{Lemma 6. For each $r, g_{r}$ is a homeomorphism of $Z$ onto $Z$.}

Proof. Let $r$ be a positive integer. It is clear that $g_{r}$ is one-to-one and onto. Let $k$ be a positive integer. There exists a $j$ such that $g_{r}\left(p_{k}\right)=p_{j}$. Suppose that $U \in N\left(p_{k}\right)$. Now there exists an $n$ and sets $U_{n}, U_{n+1}, \cdots$ such that if $s \geqq n, U_{\text {s }}$ is open in $Z_{s}, p_{k}^{s} \in U_{s}, U_{s} \widetilde{\subset} U_{s+1}$, and such that $U=\bigcup_{s=n}^{\infty} U_{s}^{*}$. Let $m$ be a positive integer such that $j \leqq 2 m$. Now since $U_{s} \widetilde{\subset} U_{s+1}$, then $U_{s=n}^{\infty} U_{s}^{*}=U_{s=m}^{\infty} U_{s}^{*}$, and hence $U=\cup_{s=m}^{\infty} U_{s}^{*}$. Now for each $s, s \geqq m, g_{r}^{s}$ is a homeomorphism and hence $g_{r}^{s}\left(U_{s}\right) \in N^{s}\left(p_{j}^{s}\right)$. Also $g_{r}^{s}\left(U_{s}\right) \widetilde{\subset} g_{r}^{s+1}\left(U_{s+1}\right)$. Now since $g_{r}\left(U_{s}^{*}\right)=\left[g_{r}^{s}\left(U_{s}\right)\right]^{*}$ and $g_{r}(U)=U_{s=m}^{\infty} g_{r}\left(U_{s}^{*}\right)$, then $g_{r}(U) \in N\left(p_{j}\right)$. This shows that $\left(g_{r}\right)^{-1}$ is continuous. Since $g_{r}=\left(g_{r}\right)^{-1}, g_{r}$ is continuous and it follows that $g_{r}$ is a homeomorphism.

The above lemmas establish the theorem.

THeOREM II. There exists a countable connected Hausdorff space $Z$ such that each point of $Z$ is a cut point of $Z$. 
Proof. Let $Y$ be a countable connected Hausdorff space and let $\left\{X_{i j}\right.$ : each of $i$ and $j$ is a positive integer $\}$ be a countably infinite family of mutually disjoint countable connected Hausdorff spaces, each disjoint from $Y$. Let $y^{1}$ be a one-to-one sequence onto $Y$, i.e., let a fixed denumeration $y_{1}^{1}, y_{2}^{1}, y_{3}^{1}, \cdots$ be placed on the elements of $Y$, and for each $(i, j)$ let $x^{i j}$ be a one-to-one sequence onto $X_{i j}$.

If $p \in Y$, then $\Re(p)$ denotes the neighborhood system of $p$ in $Y$, and if $p$ is a point of some $X_{i j}$, then $\Re(p)$ denotes the neighborhood system of $p$ in $X_{i j}$.

Let $Z_{1}$ be $\bigcup_{i=1}^{\infty}\left(X_{1 i}-\left\{x_{1}^{1 i}\right\}\right) \cup\left\{\left(y_{i}^{1}, x_{1}^{1 i}\right): i\right.$ is a positive integer $\}$. A neighborhood system for $Z_{1}$ is defined as follows: If $z \in \bigcup_{i=1}^{\infty}\left(X_{1 i}-\left\{x_{1}^{1 i}\right\}\right)$, then for some $i$ and some $k, k \neq 1, z=x_{k}^{1 i}$, and in this case $N^{1}\left(x_{k}^{14}\right)$, the neighborhood system of $x_{k}^{1 t}$ in $Z_{1}$, is $\left\{U: U \in \Re\left(x_{k}^{1 t}\right)\right.$ and $\left.x_{1}^{1 i} \notin U\right\}$. In order to define neighborhoods in $Z_{1}$ of the remaining points of $Z_{1}$, certain functions will be introduced. Suppose that $i$ is a positive integer and that $V \in \mathfrak{N}\left(y_{i}^{1}\right)$. Let $V^{*}$ be $\left\{\left(y_{i}^{1}, x_{1}^{11}\right): y_{i}^{1} \in V\right\} . V^{*}$ is a one-to-one function. Let $W(V)$ be the set of all functions $w$ on (range $V^{*}$ ) such that if $x_{1}^{1 t} \in\left(\right.$ range $V^{*}$ ), then $w\left(x_{1}^{11}\right) \in \Re\left(x_{1}^{1 i}\right)$. If $w \in W(V)$ let $U(V, w)$ be

$$
V^{*} \cup\left[\cup\left\{\left[w\left(x_{1}^{1 i}\right)-\left\{x_{1}^{1 i}\right\}\right]: x_{1}^{1 i} \in \operatorname{dom} w\right\}\right] .
$$

Now if for some $i, z=\left(y_{i}^{1}, x_{1}^{14}\right)$, then $N^{1}(z)$, the neighborhood system in $Z_{1}$ of $z$, is $\left\{U(V, w): V \in \Re\left(y_{i}^{1}\right)\right.$ and $\left.w \in W(V)\right\} . Z_{1}$ with the resulting topology is a countable connected Hausdorff space.

Lemma 1. For each $i,\left(y_{i}^{1}, x_{1}^{1 t}\right)$ is a cut point of $Z_{1}$.

Proof. Let $i$ be a positive integer. Let $A$ be $\left\{x_{j}^{11}: j>1\right\}$. Then $A$ and its complement relative to $Z_{1}-\left\{\left(y_{i}^{1}, x_{1}^{14}\right)\right\}$ are disjoint nonempty open sets whose union is $Z_{1}-\left\{\left(y_{i}^{1}, x_{1}^{11}\right)\right\}$. This establishes Lemma 1 .

Now for each $n, n \neq 1$, let $y^{n}$ be a one-to-one sequence onto $\bigcup_{i=1}^{\infty}\left(X_{(n-1) i}-\left\{x_{i}^{(n-1) i}\right\}\right)$, and let $Z_{n}$ be $\bigcup_{i=1}^{\infty}\left(X_{n i}-\left\{x_{1}^{n t}\right\}\right) \cup\left\{\left(y_{k}^{j}, x_{1}^{n k}\right)\right.$ : each of $j$ and $k$ is a positive integer and $j \leqq n\}$. A neighborhood system for $Z_{n}$ is defined as follows: If $z \in \cup_{i=1}^{\infty}\left(X_{n i}-\left\{x_{i}^{n i}\right\}\right)$, then for some $i$ and some $k, k \neq 1, z=x_{k}^{n i}$, and in this case $N^{n}\left(x_{k}^{n t}\right)$, the neighborhood system in $Z_{n}$ for $x_{k}^{n i}$, is $\left\{U: U \in \mathfrak{N}\left(x_{k}^{n i}\right)\right.$ and $\left.x_{1}^{n i} \notin U\right\}$. In order to define neighborhoods in $Z_{n}$ of the remaining points of $Z_{n}$, certain functions will be introduced. Suppose that $i$ is a positive integer and that $V \in N^{n-1}\left(y_{i}^{n}\right)$. Let $V^{*}$ be $\left\{\left(y_{j}^{n}, x_{1}^{n j}\right): y_{j}^{n} \in V\right\} . V^{*}$ is a one-to-one function. Let $W(V)$ be the set of all functions $w$ on (range $V^{*}$ ) such that if $x_{1}^{n j} \in$ (range $\left.V^{*}\right)$, then $w\left(x_{1}^{n j}\right) \in \Re\left(x_{1}^{n j}\right)$. If $w \in W(V)$, let $U(V, w)$ be $V^{*} \cup\left[\cup\left\{\left[w\left(x_{1}^{n j}\right)-\left\{x_{1}^{n j}\right\}\right]: x_{1}^{n j} \in \operatorname{dom} w\right\}\right]$. Now if for some $i, z$ 
$=\left(y_{i}^{n}, x_{1}^{n i}\right)$, then $N^{n}(z)$, the neighborhood system in $Z_{n}$ of $z$ is $\left\{U(V, w): V \in N^{n-1}\left(y_{i}^{n}\right)\right.$ and $\left.w \in W(V)\right\}$. Suppose that $i$ and $j$ are positive integers, $j \leqq n-1$, and that $V \in N^{n-1}\left(y_{i}^{j}, x_{1}^{j k}\right)$. Let $V^{*}$ be $\left\{\left(y_{s}^{r}, x_{1}^{r s}\right):\left(y_{s}^{r}, x_{1}^{r s}\right) \in V\right.$ or $\left.y_{s}^{z} \in V\right\}$. Let $W(V)$ be the set of all functions $w$ on $\left\{x_{1}^{n s}:\left(y_{s}^{n}, x_{1}^{n s}\right) \in V^{*}\right\}$ such that if $x_{1}^{n s} \in\left\{x_{1}^{n s}:\left(y_{s}^{n}, x_{1}^{n s}\right) \in V^{*}\right\}$, then $w\left(x_{1}^{n s}\right) \in \Re\left(x_{1}^{n s}\right)$. If $w \in W(V)$, let $U(V, w)$ be

$$
V^{*} \cup\left[\cup\left\{\left[w\left(x_{1}^{n s}\right)-\left\{x_{1}^{n s}\right\}\right]: x_{1}^{n s} \in \operatorname{dom} w\right\}\right] \text {. }
$$

Now if for some $i$ and some $j, j \leqq n-1, z=\left(y_{i}^{j}, x_{1}^{\sharp}\right)$, then $N^{n}(z)$, the neighborhood system in $Z_{n}$ of $z$, is $\left\{U(V, w): V \in N^{n-1}\left(y_{i}^{j}, x_{1}^{\jmath^{4}}\right)\right.$ and $w \in W(V)\}$. Now for each $n, Z_{n}$, with the resulting topology, is a countable connected Hausdorff space.

Lemma 2. For each $i, j$, and $n, i \leqq n,\left(y_{j}^{i}, x_{1}^{i j}\right)$ is a cut point of $Z_{n}$.

Proof. Lemma 1 shows that the conclusion is true if $n=1$. Let $n$ be a positive integer greater than 1 , and suppose that if $k$ is a positive integer less than $n$ and $i$ and $j$ are integers, $i \leqq k$, then $\left(y_{j}^{i}, x_{1}^{i j}\right)$ is a cut point of $Z_{n-1}$. Let $i$ and $j$ be positive integers, $i \leqq n$.

Now if $i<n$, there exist disjoint nonempty open subsets $A$ and $B$ of $Z_{n-1}-\left\{\left(y_{j}^{i}, x_{1}^{i j}\right)\right\}$ such that $A \cup B=Z_{n-1}-\left\{\left(y_{j}^{i}, x_{1}^{i j}\right)\right\}$. Let $J_{0}$ be a subset of the positive integers such that $k \in J_{0}$ if and only if $y_{k}^{n} \in A$. Let $C$ be $\left\{\left(y_{s}^{r}, x_{1}^{r s}\right):\left(y_{s}^{r}, x_{1}^{r s}\right) \in A\right.$ or $\left.y_{s}^{r} \in A\right\} \cup\left[\cup\left\{\left(X_{n k}-\left\{x_{1}^{n k}\right\}\right): k \in J_{0}\right\}\right]$, and $D$ be $\left\{\left(y_{s}^{r}, x_{1}^{r s}\right):\left(y_{s}^{r}, x_{1}^{r s}\right) \in B\right.$ or $\left.y_{s}^{r} \in B\right\} \cup\left[\cup\left\{\left(X_{n k}-\left\{x_{1}^{n k}\right\}\right): k \notin J_{0}\right\}\right]$. Now $C$ and $D$ are disjoint nonempty open subsets of $Z_{n}-\left\{\left(y_{j}^{i}, x_{1}^{i j}\right)\right\}$ such that $C \cup D=Z_{n}-\left\{\left(y_{j}^{i}, x_{1}^{i j}\right)\right\}$.

If $i=n$, the proof that $\left(y_{j}^{i}, x_{1}^{i j}\right)$ is a cut point of $Z_{n}$ is similar to the proof that $\left(y_{j}^{1}, x_{1}^{1 /}\right)$ is a cut point of $Z_{1}$, and hence is omitted.

Let $Z$ be $\left\{\left(y_{j}^{i}, x_{1}^{i j}\right)\right.$ : each of $i$ and $j$ is a positive integer $\}$. A neighborhood system for $Z$ is defined as follows: Suppose that $z \in Z$. Then for some $i$ and some $j, z=\left(y_{j}^{i}, x_{1}^{i j}\right)$. Let $S(z)$ be the set of all functions $V$ on $\{i, i+1, i+2, \cdots\}$ such that (1) $V_{i} \in N^{i}(z)$ and (2) if $k>i$, then $V_{k} \in N^{k}(z)$ and for some element $w$ of $W\left(V_{k-1}\right), V_{k}=U\left(V_{k-1}, w\right)$. Now if for some $i$ and some $j, z=\left(y_{j}^{i}, x_{1}^{i j}\right)$, then $N(z)$, the neighborhood system in $Z$ of $z$ is $\left\{\left[\cup_{k=t}^{\infty}\left(V_{k} \cap Z\right)\right]: V \in S(z)\right\} . Z$ with the resulting topology is a countable connected Hausdorff space.

Lemma 3. Each point of $Z$ is a cut point of $Z$.

Proof. Suppose that $z \in Z$. Then there exist integers $i$ and $j$ such that $z=\left(y_{j}^{i}, x_{1}^{i j}\right)$. Now there exist sequences $A_{i}, A_{i+1}, A_{i+2}, \cdots$ and $B_{i}, B_{i+1}, B_{i+2}, \cdots$ such that for every positive integer $k$ greater than $i-1$, (1) $A_{k}$ and $B_{k}$ are nonempty disjoint open subsets of $Z_{k}$ 
$-\left\{\left(y_{j}^{i}, x_{1}^{i j}\right)\right\}$ such that $A_{k} \cup B_{k}=Z_{k}-\left\{\left(y_{j}^{i}, x_{1}^{i j}\right)\right\}$, (2) if $v \in A_{k}$ and $V$ is an element of $N^{k}(v)$ such that $V \subset A_{k}$, then for every element $w$ of $W(V), U(V, w) \subset A_{k+1}$, and (3) if $v \in B_{k}$ and $V$ is an element of $N^{k}(v)$ such that $V \subset B_{k}$, then for every element $w$ of $W(V), U(V, w) \subset B_{k+1}$. Let $A$ be $\cup_{k=i}^{\infty}\left(A_{k} \cap Z\right)$ and $B$ be $\cup_{k=i}^{\infty}\left(B_{k} \cap Z\right)$. Then $A$ and $B$ are nonempty disjoint open subsets of $Z-\left\{\left(y_{j}^{i}, x_{1}^{i j}\right)\right\}$ such that $A \cup B$ $=Z-\left\{\left(y_{j}^{i}, x_{1}^{i j}\right)\right\}$.

The above lemmas establish the theorem.

THEOREM III. There exists a countable connected Hausdorff space $Z$ such that the only homeomorphism of $Z$ onto itself is the identity.

OUtLINE OF PROOF. If $Z$ is a connected space and $z \in Z$, then the statement that $z$ has component number $n$ means that $n$ is a positive integer and that $Z-\{z\}$ has exactly $n$ components. Using a construction similar to that of Theorem II, a countable connected Hausdorff space $Z$ can be constructed such that (1) every point of $Z$ is a cut point of $Z$ and (2) distinct points of $Z$ have distinct component numbers. It follows that the only homeomorphism of $Z$ onto itself is the identity.

\section{REFERENCES}

1. R. H. Bing, A countable connected Hausdorff space, Proc. Amer. Math. Soc. vol. 4 (1953) p. 474.

2. M. Brown, A countable connected Hausdorff space, Bull. Amer. Math. Soc. vol. 59 (1953) p. 367.

3. E. Hewitt, On two problems of Urysohn. Ann. of Math. (2) vol. 47 (1946) pp. 503-509.

4. P. Urysohn, Über die Mächtigkeit der zusammenhängen Mengen, Math. Ann., vol. 94 (1925) pp. 262-295.

State University of Iowa 